\title{
Positioning Accuracy in Optical Trap Assisted Nanostructuring
}

\author{
Ulf QUENTIN ${ }^{1,2}$, Karl-Heinz LEITZ ${ }^{1,2}$, Jan PAULUS ${ }^{1,2}$, Ilya ALEXEEV ${ }^{1,2}$, Michael SCHMIDT ${ }^{1,2}$ \\ ${ }^{1}$ Chair of Photonic Technologies, University of Erlangen-Nuremberg, Paul-Gordan-Straße 3, \\ 91052 Erlangen, Germany \\ ${ }^{2}$ Erlangen Graduate School in Advanced Optical Technologies, Paul-Gordan-Straße 6, 91052 \\ Erlangen, Germany \\ E-mail: ulf.quentin@lpt.uni-erlangen.de
}

\begin{abstract}
The well-known optical diffraction limit prevents laser radiation from being focused to arbitrary small spots. Utilizing spherical micro-particles as near-field lenses provides an easy and robust way to overcome this limitation and to generate nanoscale surface structures. To achieve this, microspheres are positioned and translated with an optical trap and then used to focus pulsed laser radiation. The paper at hand presents the factors which influence the positioning accuracy in optical trap assisted nanostructuring. Theoretical considerations are backed up with experimentally obtained values of the surface structure accuracy in dependence of optical trap stiffness and particle position.
\end{abstract}

DOI:10.2961/jlmn.2013.01.0016

Keywords: nanostructuring, direct-write, optical trap, ultrafast lasers

\section{Introduction}

Recently, a flexible, direct-write approach for laser nanostructuring has been presented, which uses optically trapped spherical particles as micro-lenses to focus pulsed laser radiation in order to overcome the optical diffraction limit $[1,2]$. Optical traps consist of a tightly focused $\mathrm{cw}$ laser beam which can confine micro-particles threedimensionally. With this contactless method, it is possible to position particles of some micrometres or smaller in diameter with a precision of some ten nanometres on the surface of a substrate. Irradiation of the trapped particles with pulses of a second laser (the so-called structuring laser) leads to an enhanced electro-magnetic field underneath the particle. The resulting intensity can exceed the ablation threshold of the substrate leading to nanoscale surface structures. Generated feature sizes have been smaller than $100 \mathrm{~nm}$ so far [1].

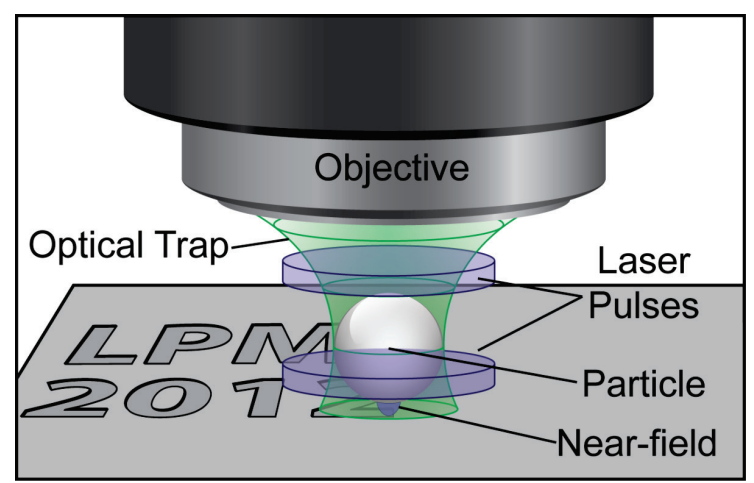

Fig. 1: Principle of optical trap assisted nanostructuring

To achieve such small dimensions, it is crucial to maintain a high positioning accuracy, not only laterally in the $\mathrm{x}-\mathrm{y}$-plane but also axially in z-direction.
In the following sections, we present theoretical considerations concerning the achievable accuracy and measurements of the particle position laterally and axially during the structuring process.

\section{Theoretical consideration}

\subsection{Forces in optical trap assisted nanostructuring}

Several forces have to be considered when pursuing a high precision in optical trap assisted nanostructuring (OTAN). They can be grouped into optical forces, surface forces, forces due to the surrounding medium and the ablation itself.

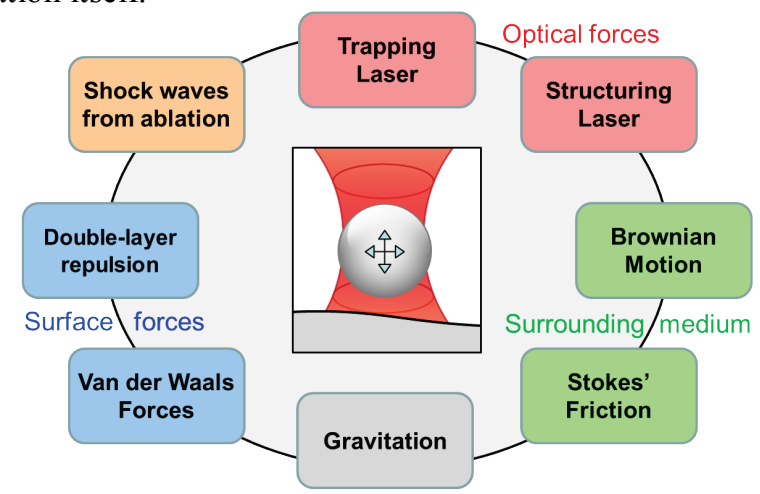

Fig. 2: Influences on the particle position

Optical forces naturally arise from both the trapping laser and the pulsed structuring laser. The optical trapping forces have been theoretically described extensively by Arthur Ashkin and others [3,4]. The stiffness of an optical trap increases with the numerical aperture (NA) of the focusing optics and is directly proportional to the laser power [3]. Typically, microscopy objectives with NA $>1.0$ are used and the laser powers are in the range of some 
ten Milliwatts. The resulting forces are on the order of Piconewtons.

In case of OTAN, the structuring laser is not focused onto the trapped particle and therefore does not add to the stiffness of the optical trap. Instead, the laser pulses lead to a net force in beam propagation direction, which does only act within the short duration of a pulse. The transferred impulses, however, can be high enough to deflect the particle or even kick it out of the optical trap, when the power of the trapping laser itself is not high enough. Although this influence can be minimized with the right settings, it is not negligible.

Another source of forces on the trapped particle is the close vicinity of the particle and the substrate: A class of surface forces, the van der Waals forces, arise from interaction between (induced) dipoles of the particle and the substrate's surface. Since these forces are in inverse proportion to the sixth power of the distance between particle and substrate, van der Waals forces do only play a role for distances of some nanometres [5]. For a distance of tens or hundreds of nanometres, which is usually the case for OTAN [2], the electrostatic double-layer repulsion is more important. Surfaces in aqueous solutions usually form two layers of negative and positive charges on and respectively near the surface [5]. When two surfaces approach, the layers of equal charges which surround both give rise to an entropic force due to repulsion of identically charged ions [5]. As the name double-layer repulsion indicates, the net force between two surfaces is generally repulsive, where the van der Waals forces can also be attractive [5].

Probably the most important influence on position accuracy results solely from the surrounding medium of the particle. Although it is theoretically possible to optically trap particles in air, it is mostly done in an aqueous solution or pure water. When moving the particle, Stokes' law for friction in viscous fluids applies and the drag force limits the maximal velocity at which the particles can be translated. More importantly, Brownian motion deflects the particle out of its equilibrium position even when the optical trap is not moved relatively to the surrounding medium.

Brownian motion denotes a stochastic force that acts on particles in a fluid due to thermal fluctuations of the fluid molecules. The theory of Brownian motion is well-known today and holds important aspects for the position accuracy in OTAN. In general, Brownian motion is more active, the smaller the particles are, the lower the fluid's viscosity is and the higher the temperature is. The motion can be described as a stochastic velocity process, the OrnsteinUhlenbeck process: Accordingly, the variance of the velocity of a particle in a viscous fluid can be described as in equation 1 [6].

$$
\operatorname{var}\left(v_{t}\right)=\beta D\left(1-e^{-2 \beta t}\right)
$$

Here, $D$ is Einstein's coefficient of diffusion

$$
D=\frac{k_{B} T}{\gamma}
$$

with Boltzmann's constant $k_{B}$, temperature $T$ and Stokes' damping coefficient $\gamma=6 \pi \eta a$, with dynamic viscosity $\eta$ and particle radius $a . \beta=\gamma / m$ with particle mass $m$.
From this, the theory of Brownian motion in a force field can be obtained [6], which is the case for an optically trapped particle in a viscous medium. It can be shown that the power spectral density $S$ of a trapped particle can be described by a Lorentzian function [7]:

$$
S(f)=\frac{D}{2 \pi^{3}\left(f_{0}^{2}+f^{2}\right)},
$$

which depends on Einstein's coefficient of diffusion $D$ and the so-called corner frequency $f_{0}$ :

$$
f_{0}=\frac{\kappa}{2 \pi \gamma}
$$

The corner frequency is directly proportional to the optical trap stiffness $\kappa$ and therefore a measure for the quality of the optical trap.

In order to obtain the stiffness, the particle position in a lateral dimension needs to be measured over time. Subsequently, a Fourier transformation and fitting of a Lorentzian curve leads to the corner frequency and thereby to the stiffness of the optical trap (see Fig. 3).

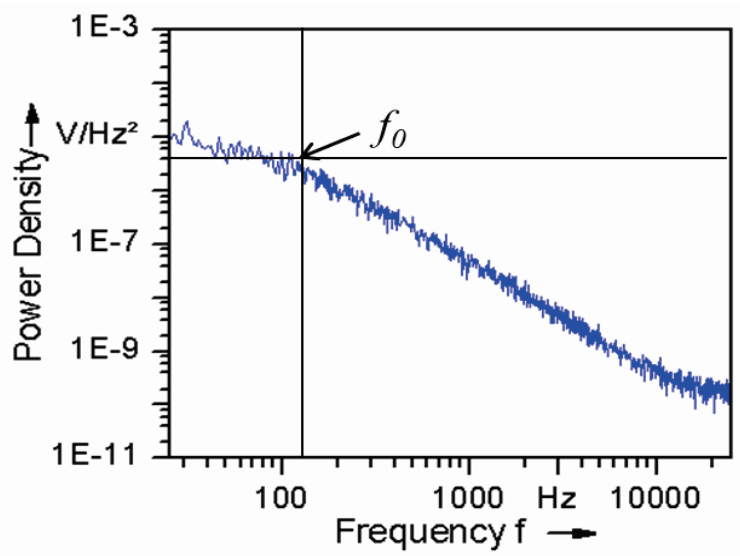

Fig. 3: Experimentally obtained power spectral density of a trapped particle position. The measurement is taken over 2 seconds and then Fourier transformed. By fitting a Lorentzian curve to the result, the corner frequency $f_{0}$ can be obtained, which is a measure for the optical trap stiffness. Methods to measure the particle position are described in the references $[7,8]$.

The mean square deflection of a trapped particle is described by equation 5 [9].

$$
\left\langle x^{2}\right\rangle=2 \pi \int_{0}^{\infty} S(f) d f
$$

Integrating the power spectral density $S$ leads to the following, very simple expression for the standard deviation $\sigma_{x}$ of the particle position [9]:

$$
\sigma_{x}=\sqrt{\frac{k_{B} T}{\kappa}}
$$

The deflection of an optically trapped particle by Brownian motion is obviously affected by the stiffness of the optical trap $\kappa$ and the temperature of the surrounding medium $T$. Plotting the standard deviation of the position vs. the optical trap stiffness leads to Fig. 4.

It can be seen that increasing the stiffness (by increasing the laser power) decreases the deflection of the particle. However, a deflection due to Brownian motion 
cannot be eliminated totally since the standard deviation of the position decreases very slowly as the stiffness is increased to more than $100 \mathrm{pN} / \mu \mathrm{m}$. With reasonable laser powers, an approximate limit of $5 \mathrm{~nm}$ for the standard deviation of the position cannot be overcome due to damage thresholds of the trapped particles and substrates. Therefore, Brownian motion ultimately limits the achievable positioning accuracy. This theory applies to all three dimensions.

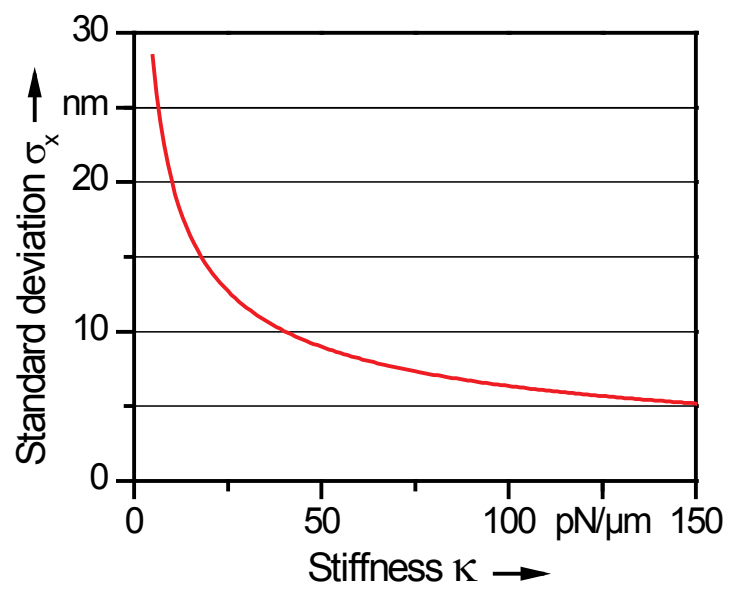

Fig. 4: Standard deviation of an optically trapped particle due to Brownian motion vs. stiffness of the optical trap at a temperature of $20^{\circ} \mathrm{C}$. The mean particle position is 0 .

\subsection{Required positioning accuracy}

In case of the lateral particle confinement, the required accuracy is obviously determined by the targeted width of the generated structures. Any deflection of the particle will add up to the smallest achievable line width. If the target width is $100 \mathrm{~nm}$, a mean deflection of $10 \mathrm{~nm}$ already leads to an increase in width of ten percent. When targeting such small dimensions, the full potential of the optical trapping system in terms of stiffness has to be realized in order to get the smallest possible position deviation (see Fig. 4).

To determine the required axial accuracy, the electromagnetic field beneath a particle has to be considered. The sensitivity of the intensity distribution in the substrate plane to axial movements of the particle defines the susceptibility of the whole OTAN process to axial deflections. To examine the resulting fields of particle focussing, we set up a simulation model in COMSOL Multiphysics. The results are depicted in Fig. 5.

When the surrounding medium is air (Fig. 5a), the enhanced field extents not further than approximately $250 \mathrm{~nm}$ (FWHM) in axial direction. In case of water, however, the dimensions of the resulting field changes significantly (Fig. 5b). The field is elongated in the axial dimension to a depth of focus of approximately $1.4 \mu \mathrm{m}$ and the field is shifted by $350 \mathrm{~nm}$ in beam propagation direction. This leads to a gap between particle and enhanced field, which prevents nanostructuring with particles that are too close to the surface so that a certain distance has to be maintained. The elongated field also leads to a need of an increased overall power of the structuring laser to exceed the ablation threshold, since the maximal intensity is decreased compared to air as the surrounding medium. On the positive side, the elongation effect makes the OTAN process less sensitive to deviation in the axial particle position. The lateral accuracy a structuring process based on a Bessel beam optical trap has already been comprehensively discussed by McLeod and Arnold [10]. a)

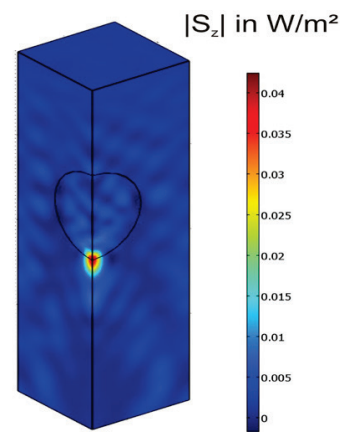

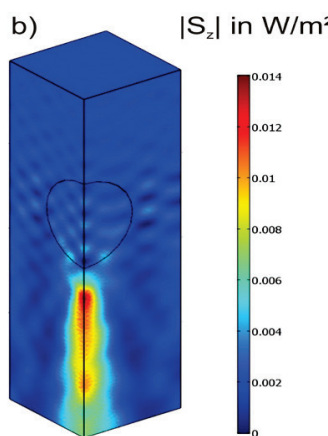

Fig. 5: Electro-magnetic field underneath a $1 \mu \mathrm{m} \mathrm{SiO}_{2}$ particle. a) Surrounding medium: air; b) water. The simulated laser wavelength is $400 \mathrm{~nm}$.

\section{Experimental setup and procedure}

In order to examine the implications of the theoretical consideration described in section 2 on the experimental results, we conducted a number of experiments of optical trap assisted nanostructuring with varying parameters like trap stiffness and axial particle position. The used setup and the measurement procedures are described below.

\subsection{OTAN setup}

The experimental setup used for OTAN experiments is shown in Fig. 6. The laser applied for optical trapping is a $\mathrm{cw}$, frequency doubled $\mathrm{Nd}$ :YAG laser with a wavelength of $532 \mathrm{~nm}$ and an output power of up to $4 \mathrm{~W}$ (Laser Quantum Finesse). Its beam is expanded by a two lens Galilean telescope before it gets focused by a water immersion objective with a numerical aperture of 1.2 (Olympus UPlanSApo). Water immersion is necessary, because the particles are diluted in water and spherical aberrations have to be avoided. The forward scattered light from a trapped particle is collected by a condenser lens and imaged onto a quadrant photodiode. This is used to measure the particle position in the lateral $x-y$ plane.

For structuring, a frequency doubled Ti:Sapphire femtosecond laser is used with a wavelength of $400 \mathrm{~nm}$ and pulse duration of $120 \mathrm{fs}$. A two lens telescope is used to create a diverging beam. This is necessary in order not to focus the pulsed laser beam onto the particle, but rather shift its focus in the axial direction and thus illuminate the particle as homogenously as possible.

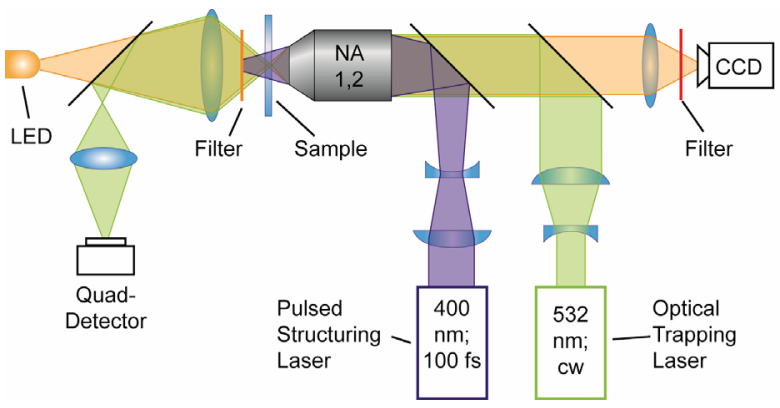

Fig. 6: Experimental setup of OTAN. Green: trapping laser, blue: structuring laser: orange: imaging 
The NA 1.2 objective is also used to image the structuring process onto a CCD camera. Therefore, the sample is illuminated by an LED with a wavelength of $570-620 \mathrm{~nm}$. In this configuration, a high-pass filter with a cut-on wavelength of $550 \mathrm{~nm}$ prevents any laser radiation from reaching the camera.

For positioning of the sample, a 6-axis stepper motor stage with 3-axis piezo actuators is used (Thorlabs Nanomax600). The accuracy of the positioning system is $5 \mathrm{~nm}$ in all dimensions.

\subsection{Measurement of the axial particle position}

The lateral position of a trapped particle can be measured either directly by evaluating the CCD camera pictures or via the quadrant photodiode (see [7] for a detailed description). Measuring the axial particle position, however, cannot be done directly without imaging from the side - which would be quite challenging in case of microparticles and a working distance of the objective of $270 \mu \mathrm{m}$.

An alternative approach of measuring the axial particle position is correlating the sizes of the imaged diffraction pattern of a particle with its position. As a particle moves in the $\mathrm{z}$ direction, its diffraction pattern changes and the diameter increases the further it is displaced from the object plane of the imaging system (see Fig. 7). Since the change in diameter increases linearly with the distance, this effect can be used to determine the particle position.

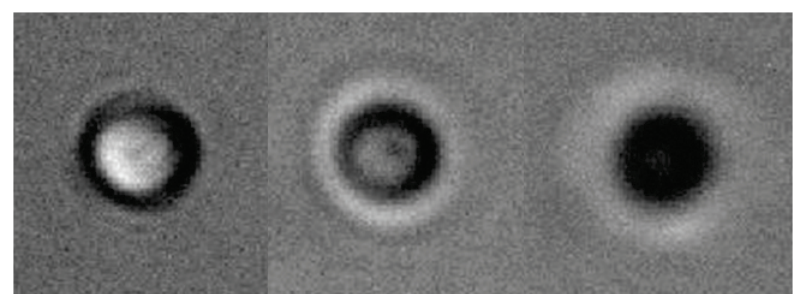

Fig. 7: Different axial particle position relative to the object plane lead to different diffraction patterns.

Our automated image analysis is done in Matlab and consists of the following steps: As we use noise sensitive gradient information for edge detection in the second step, we denoise the image first. Therefore, we apply a wavelet thresholding [12] based on an implementation of the Pattern Recognition Lab at FAU, which is available for downloading [11]. It uses the so-called double density dual-tree complex wavelet transformation [13].

In the next step, the intensity gradient magnitude image is calculated, of which the histogram is used to compute a dynamic threshold for binarization. With the isolated particle regions separated from the background, we identify their centres by their centroids. Last, the diameter has to be determined for each region. The number of pixels with a certain distance (radius) to its region centre is accumulated. This results into different scores for different radii where the radius with the highest score is classified as the diffraction pattern radius. The centre of the particle region is refined until the change in resulting diffraction diameter becomes negligible for a more stable result. The image processing pipeline for detecting the particles is illustrated in Fig. 8.

In order to use this method for measuring the position of any given particle, a calibration has to be done first. For this, a particle has to be moved relatively to the object plane in defined steps. This is done best with a particle stuck on a sample surface: the positioning system can then provide the necessary defined translation.

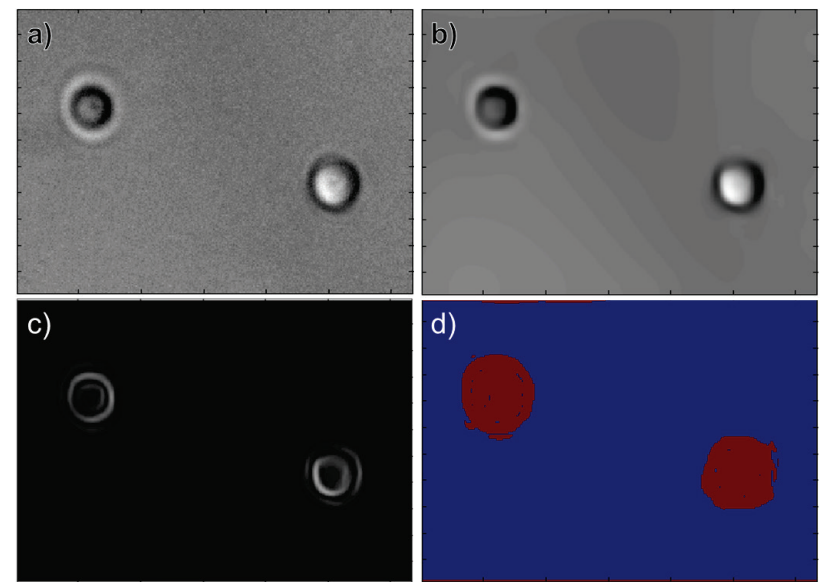

Fig. 8: Procedure of measuring the size of the diffraction pattern of a particle: a) original CCD image, b) denoised image, c) intensity gradient magnitude, d) thresholded and clustered image

As can be seen in Fig. 9, this procedure leads to the expected linear relation between measured diffraction pattern size and axial particle position.

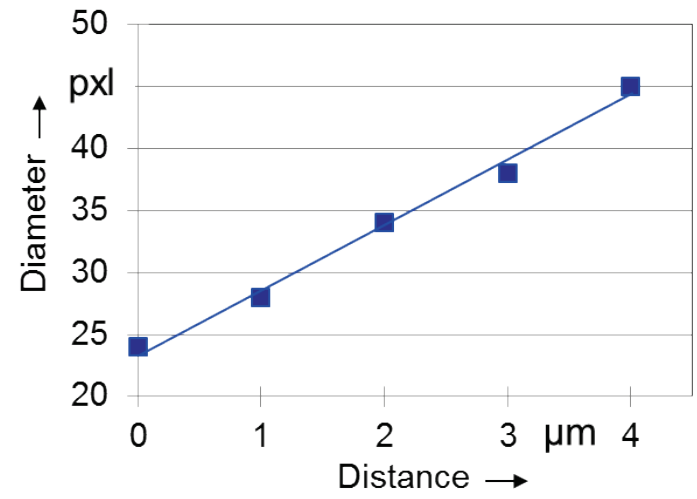

Fig. 9: Calibration curve for the axial particle position: Diameter of the diffraction pattern of a particle vs. particle distance from the object plane.

\section{Results and discussion}

In order to evaluate the influence of the optical trap stiffness on the accuracy of the structures generated by OTAN, we varied the trapping laser power (cp), which is directly proportional to the stiffness, and compared it to the resulting structures.

To do this, we took SEM pictures of several generated lines and applied the same denoising algorithm mentioned in section 3.2. The denoised pictures were rotated and for each structured line the centre pixel of each image line was identified. The standard deviation of all centre pixel positions for one structured line is then a measure for the position accuracy of the optically trapped particle during the OTAN process.

Converting theses values from pixels to nanometres results in the graph displayed in Fig. 11. The experiments 
were conducted with $2 \mu \mathrm{m} \mathrm{SiO}_{2}$ particles on a polyimide coated glass slide. The resulting structures had a width of approximately $500 \mathrm{~nm}$. The used trapping laser powers were from $150 \mathrm{~mW}$ to $500 \mathrm{~mW}$, the range that we usually use in our experiments. Increasing the power further provokes the risk of damaging the substrate with the trapping laser. The pulse repetition frequency of the structuring laser was $1000 \mathrm{~Hz}$, allowing for feed rates of $5 \mu \mathrm{m} / \mathrm{s}$.

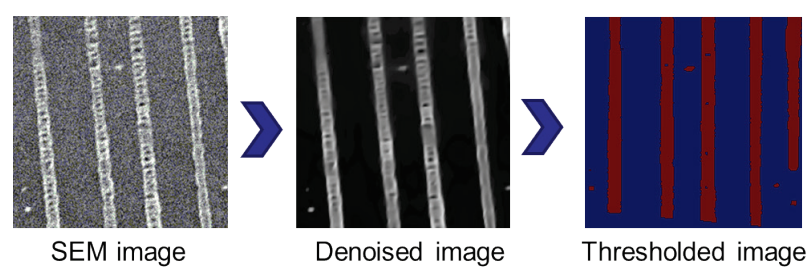

Fig. 10: Procedure to evaluate the deviation of the centre along OTAN structured lines

As can be seen in Fig. 11, the lateral position deviation of the structured lines can be decreased from approximately $110 \mathrm{~nm}$ to $30 \mathrm{~nm}$ by increasing the laser power by a factor of three. The theory of Brownian motion predicts a curvature as shown in Fig. 4. The experiments, however, revealed a linear dependence of the lateral position deviation on the optical trap stiffness (Fig. 11). Therefore, the movement of the particle cannot be explained with Brownian motion. As mentioned in section 2.1, the shock waves that are induced by the ablation underneath the particle are another important factor that influences the positioning stability. Each laser pulse leads to a shockwave that deflects the particle out of equilibrium. The exact direction and magnitude of this deflection is stochastically distributed. Since the structuring laser parameters (pulse energy and repetition rate) were kept constant, the mean acceleration of the particle is constant as well. Thus, a linear increase of the trapping laser power leads to a proportional increase in trapping stiffness and therefore to the observed linear decrease in the lateral position deviation shown in Fig. 11.

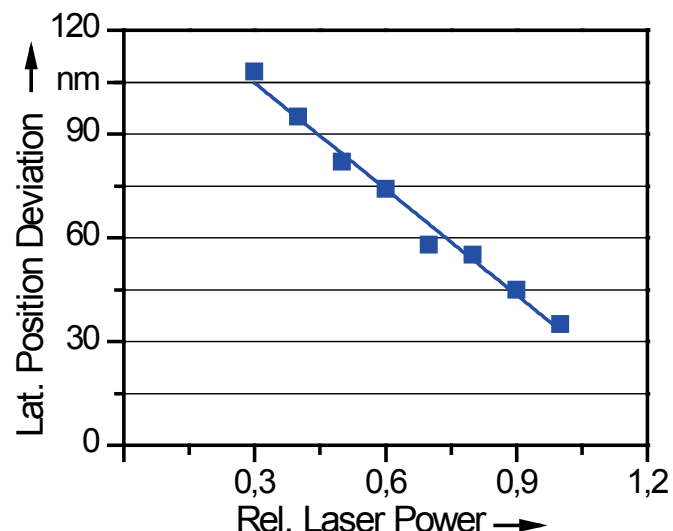

Fig. 11: Lateral deviations of the centres along structured lines in dependence of the laser power.

When processing materials with a low damage threshold such as the used polyimide, increasing the optical trap stiffness by increasing the trapping laser power is only a limited option. Alternatives would be to use an even higher numerical aperture objective or particles with a higher index of refraction like polystyrene instead of $\mathrm{SiO}_{2}$. Furthermore, a decrease of pulse frequency and feed rate would also lead to higher accuracy - at the cost of longer processing time. Also, it is rewarding to take great care when setting up the experiment and aligning the laser beam in order to create the stiffest optical trap possible with the given equipment.

In another experiment, we investigated the influence of the axial particle position on the resulting structures. Fig. 12 depicts the measured line width (blue squares) depending on the distance between trapped particle and substrate. For comparison, a second graph shows the simulated width at which the electric field exceeds a level corresponding to the ablation threshold (red line).

With a distance between one and two micrometres, homogeneous structures could be achieved in the experiments. A further increase in distance led to an unstable process and very irregular structuring results which could not be quantified. With a distance smaller than $1 \mu \mathrm{m}$, no ablation could be achieved. Therefore, Fig. 12 covers only a distance range of approximately one micrometre. All parameters other than the distance were kept constant.

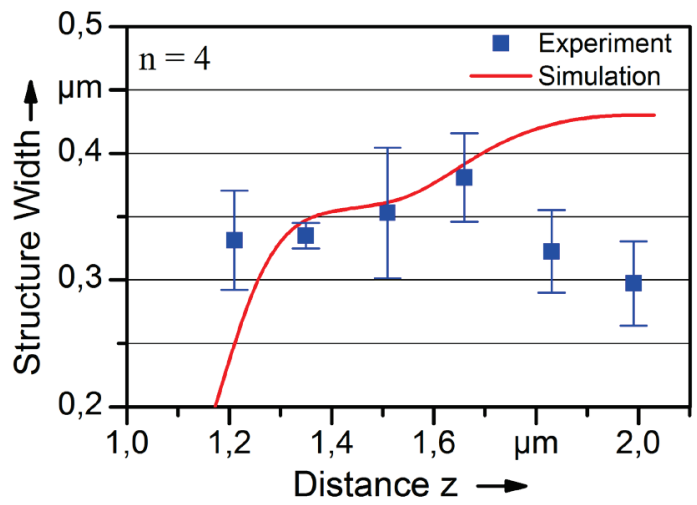

Fig. 12: Line widths of generated structures depending on the distance of the particle to the surface compared a simulation. Applied were $\mathrm{SiO}_{2}$ particles with a diameter of $2 \mu \mathrm{m}$. The structuring laser had a wavelength of $400 \mathrm{~nm}$ and a pulse duration of $100 \mathrm{fs}$.

The experiment showed a correlation between axial particle position and structure width. The maximal width at a distance from particle to substrate of $1.55 \mu \mathrm{m}$ indicates the strongest electro-magnetic field intensity at this point, i.e. at this distance the focus of the micro-lenses is approximately on the surface of the substrate. Increasing or decreasing the distance from this point leads to a decrease in structure size. The decrease of line width when the particle approaches the surface can be explained by the well-known threshold effect. As the irradiated area beneath the particle gets larger, the intensity exceeds the ablation threshold on a smaller area. Theoretically, the structure width should smoothly converge to a minimum which is reached when the particle touches the substrate, as can be seen in the simulation result in Fig. 12. Experimental constraints in accuracy, however, do not allow measurement of this, since at distances below $1 \mu \mathrm{m}$ no ablation could be observed. The simulation model predicts a further increase in structure size at distances larger than 
$1.55 \mu \mathrm{m}$. Yet the experimentally obtained values decrease after that point. At those distances, the water below the particle is irradiated with a fairly high intensity that can already alter its optical properties leading to a field distribution that differs significantly from the calculated values.

Unfortunately, the experimental data show rather high standard deviations of up to $15 \%$. A closer look into our simulation model provides a possible explanation. As can be seen in Fig. 13, the field intensity underneath a $2 \mu \mathrm{m}$ $\mathrm{SiO}_{2}$ particle irradiated with $400 \mathrm{~nm}$ fluctuates very much depending on the distance in beam propagation direction $\mathrm{z}$. Thus, small deviations in the axial position of the particle lead to high deviations in structure size. Such oscillations have also been reported by similar simulations $[14,15]$. The very irregular surface structures, which were observed experimentally for distances larger than $2 \mu \mathrm{m}$, could be evidence towards such behaviour as well. Fluctuations in the axial particle position in this range would then lead to the effect that surface structures could not be realized continuously and the line width would vary as well.

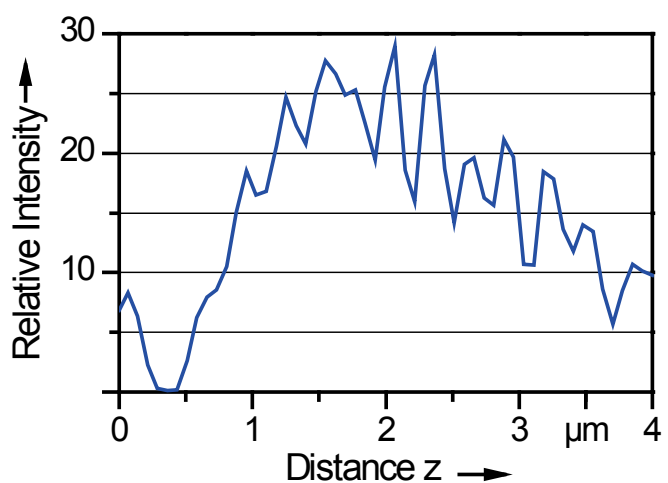

Fig. 13: Simulated relative intensity underneath a $2 \mu \mathrm{m}$ $\mathrm{SiO}_{2}$ particle irradiated with a wavelength of $400 \mathrm{~nm}$.

\section{Conclusion and outlook}

Several factors influence the position accuracy in optical trap assisted nanostructuring. Probably the single most important is Brownian motion, since it limits the practically achievable accuracy to approximately $5 \mathrm{~nm}$ in all three dimensions. To reach this limitation, a very stiff optical trap is necessary. This is important especially for the lateral accuracy, since deflections due to Brownian motion directly add to the minimal achievable width of surface structures. Our experiments have shown that structuring with a relatively high repetition rate of $1000 \mathrm{~Hz}$ allow for high feed rates of several $\mu \mathrm{m} / \mathrm{s}$. With these settings, however, the accuracy is significantly decreased by shock waves underneath the particle which deflect it from its equilibrium. Increasing the trapping laser power is the first option to decrease these deflections, but is limited due to the increased energy input which bears the risk of damaging the substrate. Therefore, increasing the optical trap stiffness by other means such as increasing the numerical aperture of the focusing optics or changing the particle material to a higher index of reflection can be preferable.

The results of optical trap assisted nanostructuring depend strongly on the axial particle position. The distance of a trapped particle to the substrate can be measured by examining the diffraction pattern of a particle imaged onto a camera. The diameter of this diffraction pattern correlates linearly with the particle position which allows for an easy calibration.

In our experiments, the distance of $2 \mu \mathrm{m} \mathrm{SiO}$ particles to the surface was between $1 \mu \mathrm{m}$ and $2 \mu \mathrm{m}$. The widths of the structured lines varied by approximately $12 \%$ over a range of $1 \mu \mathrm{m}$ in $\mathrm{z}$ direction. However, regular and constant structuring was possible throughout this range. A further increase or decrease of the distance led to irregular or no structuring at all. For smaller distances, this is in good agreement with the theory. For larger distances $(>2 \mu \mathrm{m})$, the irregularities could be accounted for by strong axial fluctuations of the intensity beneath a particle, which are predicted by our simulation model. To proof this, however, additional experiments are necessary and will be conducted in the future.

\section{Acknowledgments}

The authors gratefully acknowledge funding of the project within the priority programme 1327 "Optisch erzeugte Sub-100-nm-Strukturen für biomedizinische und technische Applikationen" and the funding of the Erlangen Graduate School in Advanced Optical Technologies (SAOT) by the German National Science Foundation (DFG).

\section{References}

[1] E. McLeod and C.B. Arnold: Nature Nanotechnology, 3, 2008, p. 413-417.

[2] U. Quentin, K.-H. Leitz, I. Alexeev and M. Schmidt: Journal of Laser Micro/Nanoengineering, 7, 2, 2012, p. 143-146.

[3] A. Ashkin: Biophysical Journal, 61, 2, 1992, p. 569 582.

[4] A. Mazolli, P.A. Maia Neto and H.M. Nussenzveig: Proceedings of the Royal Society A: Mathematical, Physical and Engineering Sciences, 459, 2040, 2003, p. 3021-3041.

[5] J.N. Israelachvili: Intermolecular and Surace Forces. Amsterdam: Elsevier, 2011.

[6] Nelson, Edward: Dynamical Theories of Brownian Motion, 2001 [cited 2011 Jul 25]. URL:http://www. math.princeton.edu/ nelson/books.html.

[7] K. Berg-Sørensen and H. Flyvbjerg: Rev. Sci. Instrum, 75, 3, 2004, p. 594.

[8] D.C. Appleyard, K. Y. Vandermeulen, H. Lee, M. J. Lang: Am. J. Phys., Vol. 75, No. 1, 2007

[9] K. Svoboda and S.M. Block: Annu. Rev. Biophys. Biomol. Struct, 23, 1, 1994, p. 247-285.

[10] E. McLeod and C. B. Arnold: Optics Express, 17, 5, 2009, p. 3640-3650

[11] Pattern Recognition Lab, FAU Erlangen-Nürnberg: Denoising Algorithms Matlab Code [cited 2012 Jun 4].URL:http://www5.informatik.uni-erlangen.de/re search/software/idaa/.

[12] S. Mallat: A Wavelet Tour of Signal Processing. Burlington: Academic Press, 2009.

[13] I.W. Selesnick: IEEE Transactions on Signal Processing, 52, 5, 2004, p. 1304-1314.

[14] J. Kofler, N. Arnold: Phys. Rev B, 73, 235401 (2006) 